\title{
Reliable Routing for Roadside to Vehicle Communications in Rural Areas
}

\author{
Shen Wan, Jian Tang and Richard S. Wolff
}

\begin{abstract}
The demands for vehicular Internet access are proliferating. Access Points (APs) can be deployed along the roadside to provide wireless coverage and network access for mobile vehicles. However, high mobility may cause frequent link breakages, which will seriously impact Quality of Service (QoS). In this paper, we study reliable routing for Roadside to Vehicle $(\mathrm{R} 2 \mathrm{~V})$ communications in rural areas where rough terrain poses additional challenges. We propose a novel routing protocol where the stationary APs play a key role in route maintenance. The protocol includes a prediction algorithm which can predict the lifetimes of wireless links with consideration for terrain effects, as well as routing algorithms which can find stable paths for packet forwarding based on the prediction. Simulation results based on OPNET Modeler and the rural roadways in the Yellowstone National Park show that the proposed protocol substantially outperforms existing ad-hoc routing protocols.
\end{abstract}

Index Terms- Vehicular ad-hoc network, roadside to vehicle communication (R2V), routing, prediction, terrain, QoS.

\section{INTRODUCTION}

Vehicular Internet access is highly desirable because it will make travel safer and more comfortable. With Internet access, passengers can obtain critical safety information such as accident warnings and road condition reports, retrieve travel related information such as weather forecasts and hotel availability, and enjoy all other traditional Internet applications.

In urban areas, conventional wireless communication infrastructures such as cellular networks are readily available and can be used to provide network access. Research projects such as COMCAR [2] and DRiVE [5] have examined methods of using existing cellular infrastructure combined with new wireless technologies to achieve Vehicle to Vehicle (V2V) and Roadside to Vehicle (R2V) communications and Internet access in urban areas. However, it is well known that cellular networks suffer from limited bandwidth, and moreover, in rural areas there is almost no fixed communication infrastructure available. The coverage provided by wireless carriers is predominantly in urban areas and along major highways. However, $78 \%$ of the total roadway miles in the U.S. are in rural areas $(3,084,000$ miles) and $60 \%$ of crash fatalities occur on rural highways (23, 876 fatalities in 2000) [4].

Wireless Access Points (APs) can be deployed along the roadside to provide Internet access for mobile vehicles. The mature 802.11-based WLAN technology [1] is an attractive solution since low-cost, off-the-shelf 802.11-based wireless routers and Network Interface Cards (NICs) can be readily

This research is funded in part by NSF grants CNS-0519403 and CNS0721880 .

Shen Wan and Jian Tang are with the Department of Computer Science at Montana State University, Bozeman, Montana 59717-3880. Email: \{swan, tang\}@cs.montana.edu. Richard Wolff is with the Department of Electrical and Computer Engineering at Montana State University, Bozeman, Montana 59717-3780. Email: rwolff@ montana.edu. used in R2V communications. However, the major weakness of 802.11 radio is its very limited transmission range, which is typically $200-300$ meters [1]. To cover wide areas such as highways in rural and remote areas, a large number of APs would be needed, resulting in a very high deployment cost. A feasible solution is to place a small number of APs along the roadside and form an ad-hoc network among vehicles to relay packets for vehicles which are out of the AP range, as illustrated in Fig. 1. In such a hybrid Vehicular Ad-hoc NETwork (VANET), high mobility may cause frequent link breakages, which will have a serious impact on the Quality of Service (QoS) of Internet access. In addition, terrain introduces new challenges for wireless communications in rural areas. Vehicles moving along rural highways may occasionally lose Line Of Sight (LOS) to neighbors or to APs due to the curving roadways and mountains, resulting in poor signal strength and intermittent connectivity.

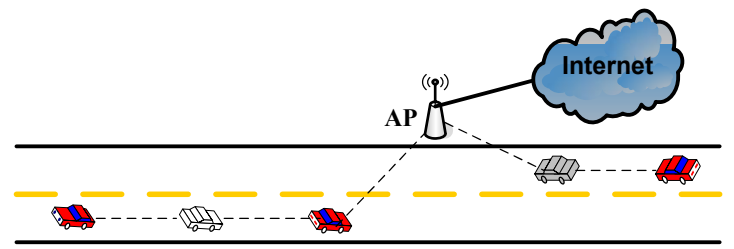

Fig. 1. Roadside to vehicle communications

In this paper, we propose a novel routing protocol to maintain reliable connections between roadside APs and vehicles on rural roadways. Specifically, we present an algorithm to predict the lifetimes of wireless links in a multi-hop network based on mobility parameters such as speed and direction, as well as the local terrain. Based on the prediction, we design two routing algorithms to find stable paths for packet forwarding. In the protocol, APs perform route maintenance by proactively replacing the current unstable routes with new routes that have longer lifetimes. In this way, service disruption can be minimized and the packet delivery ratio can be improved, which are both demonstrated by our simulation results. Our simulations were conducted using OPNET modeler [10], and the test scenarios were based on the rural roadways and terrains in the Yellowstone National Park. To our best knowledge, we are the first to study $\mathrm{R} 2 \mathrm{~V}$ communications in the context of rural areas and propose an efficient routing protocol.

The rest of this paper is organized as follows. We discuss related work in Section II. In Section III, we describe the proposed routing protocol. Numerical results are shown in Section IV and the paper is concluded in Section V. 


\section{RELATED WORK}

VANETs have recently attracted substantial attention. Protocols have also been proposed for information dissemination in VANETs. In [11], the authors defined a message propagation function that encodes information about both target areas and preferred routes. They showed how this function can be exploited in several routing protocols and evaluated the effectiveness of their approach via simulation. Several vehicleassisted data delivery (VADD) protocols were proposed to forward packets to the best road with the lowest data delivery delay [16].

V2V communication has been extensively studied [6], [7], [8], [9]. In [8], the authors evaluated the performance of a reactive routing protocol (AODV) and a geographic routing protocol (GPSR) based on realistic vehicular traces. They also presented the Preferred Group Broadcasting (PGB) strategy and the Advanced Greedy Forwarding (AGF) technique to enhance the performance of reactive and geographic routing respectively. A position-based connectivity aware routing protocol has recently been presented for $\mathrm{V} 2 \mathrm{~V}$ communications [9]. In [7], the authors proposed a prediction-based routing protocol to support the communications between mobile Internet gateways and vehicles. Lochert et al. [6] considered VANET routing in a city environment and presented a position-based routing protocol. However, ad-hoc routing for $\mathrm{R} 2 \mathrm{~V}$ communications has not been well addressed before, especially in the context of rural areas.

\section{The Proposed Routing Protocol}

In this section, we first give an overview of the proposed routing protocol. Then we describe the prediction algorithm and routing algorithms in detail.

We consider a hybrid VANET composed of vehicles constrained to move on roadways and APs deployed sparsely along the roadside. Each vehicle is equipped with a radio transceiver and a GPS receiver. Therefore, the vehicles are fully aware of their locations and mobility parameters such as speed and direction at all times. Each stationary AP is directly connected to the Internet by high capacity cables. We assume that information can be exchanged among APs via the wired network with a very short delay. We are only concerned with unicast communications between the Internet and vehicles. In addition, both APs and vehicles are assumed to transmit at the same fixed power level with transmission range $R$.

When a vehicle is out of transmission range of an AP or terrains block their communications, other vehicles will be used to relay the data traffic. Whenever a vehicle sends or relays a packet to the Internet, it piggybacks its current location and mobility information, and the corresponding timestamp in the packet. In this way, an AP can obtain location and mobility information of all vehicles in the area. Moreover, by exchanging this information among APs via the wired network, each AP can be aware of all vehicles in a wide region.

Each AP constructs a weighted communication graph $G(V, E)$, where $V=V^{\prime} \cup\{z\}$, and updates it based on the location and mobility information piggybacked in the received packets. Each node $x \in V^{\prime}$ corresponds to a vehicle or an
AP, and there is an undirected link $e=(x, y)$ between nodes $x \in V^{\prime}$ and $y \in V^{\prime}$ if either $x$ or $y$ corresponds to a vehicle and $\operatorname{dist}(x, y) \leq R$ and there is LOS between nodes $x$ and $y$, where $\operatorname{dist}(\cdot)$ returns the corresponding Euclidean distance. Note that whether there is LOS between a pair of nodes can be determined based on their locations and the related terrain information provided by U.S. Geographic Survey (USGS) maps [13]. There is no need to have a link between two nodes that are APs. The weight of a link between two vehicles or a link between a vehicle and an AP is set to its lifetime, which is predicted by the scheme presented in III-A. Node $z$ is a special sink node that corresponds to the Internet. There is a link between $z$ and every node $x \in V^{\prime}$ which corresponds to an AP and its weight is set to $\infty$.

When a vehicle has data to send to the Internet and does not have a cached route, it first initiates the route discovery process by flooding the network with Route REQuest (RREQ) packets, as the route discovery procedure used in the Dynamic Source Routing (DSR) protocol [3]. When an RREQ packet is received by an AP, the AP selects a route for the source node using the algorithm presented III-B, based on the weighted communication graph that it maintains, and signals the other APs that this route request has been processed. The AP selected by the routing algorithm will then include the computed route in a Route REPly (RREP) message and return it to the source node. The route selected by the AP is not necessarily the route contained in the RREQ packet. In addition, the new route selected by our routing algorithm may go through another AP (not the AP where the routing algorithm is executed), because the communication graph $G$ usually includes multiple APs in the area.

Our protocol is different from DSR, where the Route ERRor (RERR) based operation will be invoked to fix a broken route whenever there is a link breakage. Proactive route maintenance will be conducted periodically by the APs in our protocol. Specifically, for each flow, the corresponding AP (i.e., the AP which is used to relay packets to the Internet for this flow) executes the routing algorithm in Section III-B every $t_{m}$ seconds (the route maintenance interval), which can be set to the multiple of the mean packet arrival interval. If the path computed by the routing algorithm $P$ is different from the current path $P_{\text {cur }}$, an unsolicited RREP including new path $P$ will be sent to the source node $s$. When node $s$ receives this RREP message, it will begin to use the new path to transmit data packets. Our simulations indicate that the probability of route breakage is significantly reduced by our proactive route maintenance approach because a better route is usually selected to replace the current route before it breaks in most instances. It is possible that the current route could still break before the new route is selected and used. This might be caused by various factors such as imprecise link lifetime prediction or control packet loss. If so, the RERRbased approach specified in DSR will be employed to fix the broken route.

\section{A. The Prediction Algorithm}

It is relatively easy to predict future locations of vehicles in a VANET where mobility is restricted to roadways. Furthermore, 
vehicle speed cannot exceed the prescribed roadway segment speed limit. Terrain may also affect the communication link lifetime, so we take terrain into account for the link lifetime prediction.

We present Algorithm 1 to predict the lifetime of link $(x, y)$. The inputs of the algorithm include the current locations of nodes $x$ and $y$ denoted as $\mathbf{p}_{\mathbf{x}}$ and $\mathbf{p}_{\mathbf{y}}$, and their velocities (speeds and directions) denoted as $\mathbf{v}_{\mathbf{x}}$ and $\mathbf{v}_{\mathbf{y}}$. In addition, $\mathbf{p}_{\mathbf{x}}^{\prime}$ and $\mathbf{p}_{\mathbf{y}}^{\prime}$ represent nodes $x$ 's and $y$ 's locations after $t$ time units (prediction intervals), respectively. In the prediction, we simplify node mobility patterns by assuming that a vehicle moves at current speed and direction in the future. The lifetime of the link will be returned in terms of time units. The smaller the prediction interval, the more precise the prediction becomes.

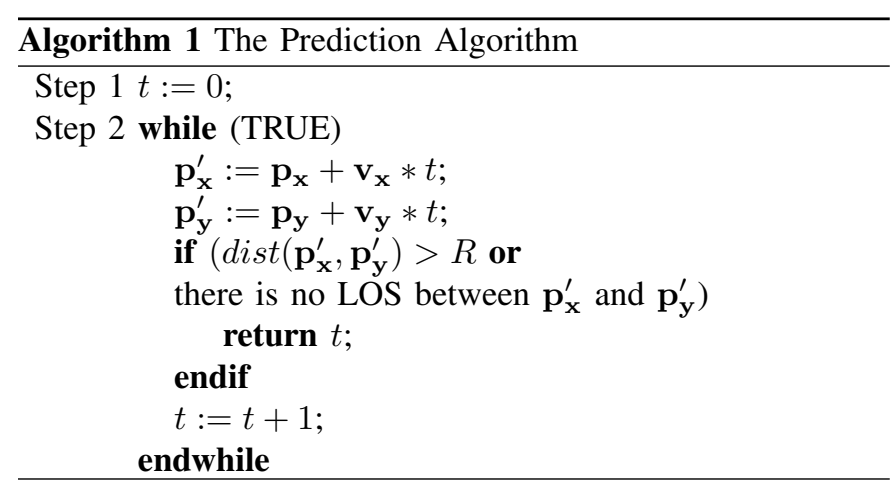

The inter-node LOS check can be done using the terrain information provided by USGS maps [13]. Although wireless communications between a pair of nodes might still be successful even if there is no LOS link, we take a conservative approach here to guarantee high reliability.

The precision of the prediction can be further improved if both APs and vehicles are pre-loaded with digital maps and the routes of the current trips are pre-computed using the map software. In this case, each vehicle is aware of its current and future locations, speeds and moving directions, which can be piggybacked in the packets sent to APs. We can then use the actual mobility information to make precise prediction of the link lifetimes. However, a drawback of this approach is that the APs need to collect extra information from each vehicle, which will increase the control overhead and, more importantly, may result in serious security and privacy issues if an AP is compromised.

\section{B. Routing Algorithms}

In this section, we present two routing algorithms which can be used by the AP to select a route for packet forwarding.

Let $e_{1}, e_{2}, \ldots, e_{p}$ be the links of a path $P$. Then the lifetime of path $P$ is $T(P)=\min _{1 \leq j \leq p} T\left(e_{j}\right)$, where $T(\cdot)$ gives the link lifetime predicted by Algorithm 1. Our routing algorithms are formally presented as Algorithm 2 and Algorithm 3. In these algorithms, $P_{\text {cur }}$ is the path currently used for packet forwarding, which is initially empty.

In order to determine which path is the "best", two factors need to be considered, the path length (the number of hops

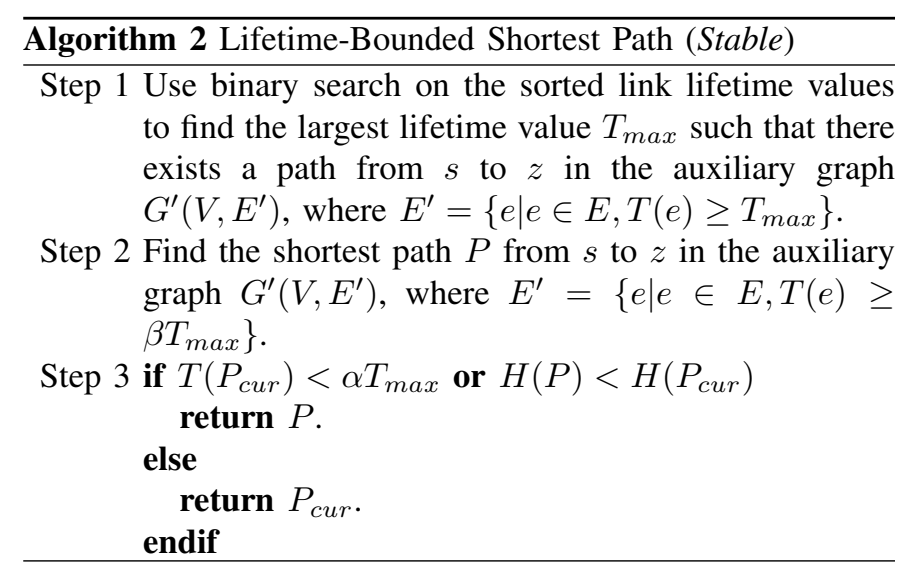

\begin{tabular}{l}
$\overline{\text { Algorithm } 3 \text { Length-Bounded Max Lifetime Path (Short) }}$ \\
\hline Step 1 Find the shortest path $P_{\text {shortest }}$ from $s$ to $z$ and its \\
length $H_{\text {min }}$.
\end{tabular}

Step 2 Use binary search on the sorted link lifetime values to find the largest lifetime value $T_{\max }$ such that there exists a path $P$ from $s$ to $z$ with $H(P) \leq\left\lceil\gamma H_{\text {min }}\right\rceil$ from $s$ to $z$ in the auxiliary graph $G^{\prime}\left(V, E^{\prime}\right)$, where $E^{\prime}=\left\{e \mid e \in E, T(e) \geq T_{\max }\right\}$.

Step 3 return $P$.

along the path) and the path lifetime. Intuitively, a path with a long lifetime may consist of a large number of short links with relatively long predicted lifetimes. If we select such paths for packet forwarding, we may end up with long end-to-end delay and high overhead. Moreover, the selected path may actually have a short lifetime because the prediction may not be very accurate, and the large path length increases the chance of link breakage. On the other hand, shortest (minimum hop-count) paths with long links may break very quickly, as mobility may cause the end nodes of a long link to move out of the transmission range quickly. Therefore, we try to achieve a good tradeoff between path lifetime and path length. Algorithm 2 finds a lifetime-bounded shortest path. In the algorithm, $\alpha$ and $\beta$ are parameters that can be set to any value in $[0,1] . H(\cdot)$ gives the path length. Note that we replace the current path with the newly computed path only if the current path's predicted lifetime drops below a threshold or a path with the same lifetime guarantee but smaller path length can be found. In this way, we can reduce the control overhead. With larger $\alpha$, path replacement will occur more frequently. Algorithm 3 finds a length-bounded maximum lifetime path. In the algorithm, $\gamma$ is a parameter that can be set to any value in $[1, \infty)$. Larger $\gamma$ will result in better lifetimes but larger path lengths.

The proposed routing algorithms are both very timeefficient. The shortest (minimum hop count) path on a graph can be easily found by the Breadth First Search (BFS) algorithm within $O(m)$ time, where $m$ is the number of links in the original communication graph $G$. The running times of Algorithm 2 and 3 are dominated by their Step 1 and Step 2 respectively, which can both be done within $O(m \log m)$ time. 


\section{PERFormance EVAluation}

In this section, we evaluate the performance of the proposed protocol using OPNET Modeler [10]. Simulations were conducted on two roadway networks in the Yellowstone National Park. The first includes a smooth terrain section of roadways near Canyon Junction, which is shown by Fig 2. The second is a rough terrain section of the Grand Loop Road, which is in a valley near the Grand Canyon of the Yellowstone River. The terrain data were acquired from USGS maps [13] and the roadway information was obtained from Yahoo! Maps [14].

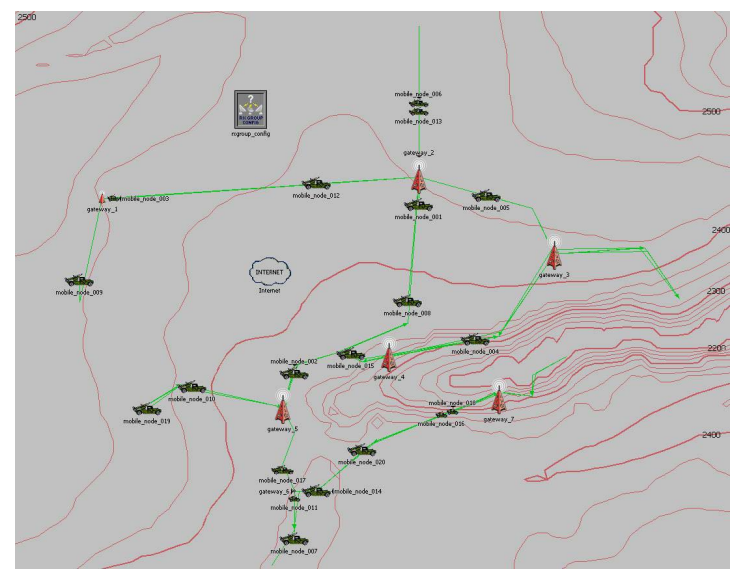

Fig. 2. Map of roadways and terrains near the Canyon Junction

In our simulations, we used the free space propagation model in the OPNET TMM (Terrain Modeling Module). The mobility model presented in [15] was applied to generate a trajectory for each vehicle, where the starting positions, the directions, and the speeds were randomly selected. A vehicle's speed was set to a random value uniformly distributed in $[0.8 \bar{v}, 1.2 \bar{v}]$, where $\bar{v}$ is the average speed. A vehicle moves along the given roadway and randomly selects a new direction at each intersection. In each simulation run, all vehicles randomly generated UDP data packets with the mean size of 1024 bits. The packet arrival follows the Poisson process and the mean packet inter-arrival rate was set to 2 seconds. The simulation settings are summarized in Table I.

TABLE I

SiMULATION PARAMETERS

\begin{aligned} & \hline \hline Total roadway length $17.5 \mathrm{~km} \\ &$ Transmission range $500 \mathrm{~m} \\ &$ Antenna height $2 \mathrm{~m} \\ &$ Number of APs 7 \\ & Average speed $(\bar{v}) 43 \mathrm{mph} \\ &$ Simulation time $6 \mathrm{~min} \\ &$ Link data rate $1 \mathrm{Mbps} \\ &$ Data packet inter-arrival time $2 \mathrm{sec}$, exponential distribution \\ & Data packet size $1024 \mathrm{~b}$, exponential distribution \\ & Mobility prediction interval $0.1 \mathrm{sec} \\ &$ Route maintenance interval $2 \mathrm{sec} \\ & \alpha 0.5 \\ & \beta 0.8 \\ & \gamma 1.5 \\ &$\hline \hline\end{aligned}

We performed simulations on VANETs with 7 APs and vehicles ranging from 20 to 160 . We compare our protocol with DSR in terms of three commonly used performance metrics: packet delivery ratio, control overhead, and average packet delay. The results are presented in Figs 3 and 4. Each number presented in the figures is the average over 10 simulation runs. In each run, different trajectories were randomly generated for all vehicles. Note that in the figures, Stable and Short are used to represent Algorithm 2 and 3 respectively. In addition, the average packet delays (y axis in Figs. 3(c) and 4(c)) are graphed using a logarithmic scale, which enables the presentation of delays ranging over several orders of magnitude. We make the following observations:

(1) Compared to DSR, our protocol (with the Stable routing algorithm) improves the data packet delivery ratio by $18.4 \%$, reduces the control overhead by $16.9 \%$, and decreases the average packet delay by $47.2 \%$, on average. Our protocol outperforms DSR in most cases, especially in relatively dense networks. Our protocol always selects stable paths for packet forwarding and proactively replaces the current path with a more stable path, significantly reducing the probability of link breakage. Therefore, packet loss is reduced accordingly. Moreover, our protocol reduces the control overhead because it does not need to send a large number of RREQ and RERR packets to fix route breakages. In addition, in DSR, when a link is broken, the nodes need to buffer the packets for the affected flow and wait for a new route to be established, which introduces a long delay. Our protocol substantially improves average packet delay by reducing the link breakage probability. A sparse network has few alternative routes (possibly only one) between a source-destination pair, which limits the improvement capability of our protocol. In this case, the performance obtained by our protocol is very close to DSR.

(2) Terrain has considerable impacts on network performance. Compared with the smooth terrain scenario (Fig. 3), rough terrains lead to a $9.9 \%-22.8 \%$ reduction in the packet delivery ratio and a $2.2 \%-24.0 \%$ increase in the control overhead. This is because rough terrain will cause more frequent link breakages. However, the simulation results indicate that our protocol consistently outperforms DSR in both scenarios.

(3) The number of vehicles (network size) affects the performance of both DSR and our protocol. With very few vehicles on the roadways, the network is frequently disconnected, which results in a low delivery ratio. However, for packets that are successfully delivered, the number of hops in the route is always very small. Therefore, the delay is short. As the number of vehicles increases, more routes between source nodes and APs become available. As a result, the packet delivery ratio increases. With the increase of network size, the delay increases rapidly, because a denser network has heavier traffic, longer MAC queues, and more collisions/retransmissions. The DSR control overhead increases sharply with the network size. However, our protocol offers a relatively constant overhead, as it takes advantage of the availability of more possible paths in a denser network and wisely selects stable paths for routing. Consequently, the number of control packets used for route repair will not be very large. However, the availability of more possible paths does not help DSR, as it may still choose "bad" links (i.e., the links which may break very soon in the future) 


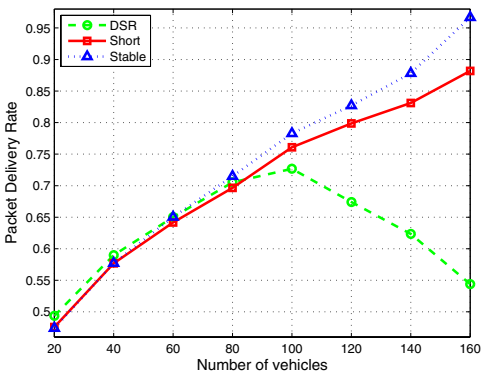

(a) Packet delivery ratio

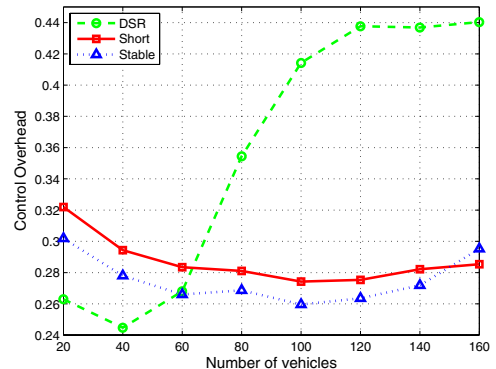

(b) Control overhead

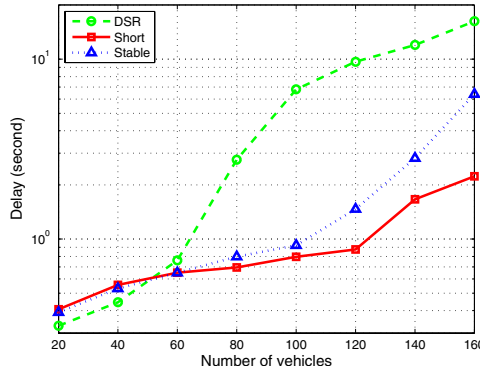

(c) Average packet delay

Fig. 3. R2V communications near the Canyon Junction (smooth terrain)

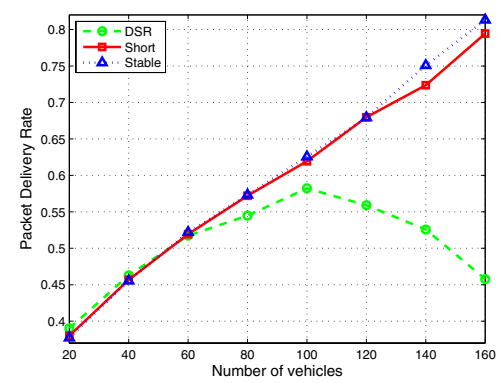

(a) Packet delivery ratio

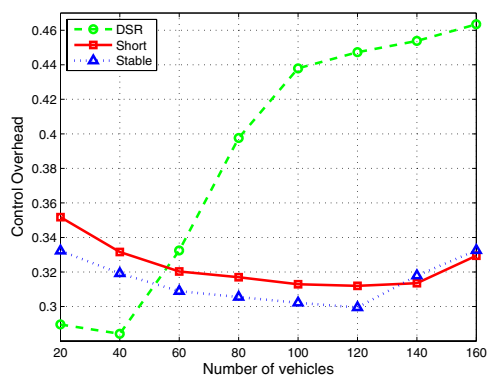

(b) Control overhead

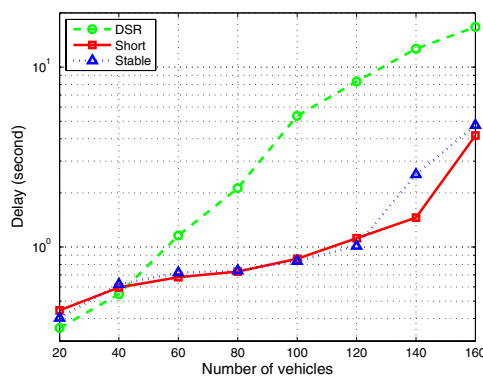

(c) Average packet delay

Fig. 4. R2V communications on the Grand Loop Road (rough terrain)

for routing. Also, in a large network, the heavy traffic demands lead to high control overhead.

(4) Our two routing algorithms, Stable (Algorithm 2) and Short (Algorithm 3), exhibit very similar performance in all the scenarios tested.

\section{CONCLUSIONS}

In this paper, we proposed a routing protocol to provide reliable R2V communications suitable for rural areas. The protocol includes a novel link lifetime prediction algorithm which takes terrain effects into account, as well as two routing algorithms which can find stable routes for packet forwarding. Using OPNET Modeler, we performed simulations to evaluate the performance of the proposed routing protocol based on the rural roadways and terrains in the Yellowstone National Park. The simulation results show that on average, our routing protocol outperforms DSR by $18.4 \%$ in terms of packet delivery ratio, by $16.9 \%$ in terms of control overhead, and by $47.2 \%$ in terms of average packet delay.

\section{REFERENCES}

[1] IEEE 802.11 Working Group, Wireless LAN Medium Access Control (MAC) and Physical Layer (PHY) Specifications, IEEE Standard, 1997.

[2] COmmunication and Mobility by Cellular Advanced Radio (COMCAR), Available at $h t t p: / / w w w . c o m c a r . d e /$

[3] D. B. Johnson, Y. Hu and D. A. Maltz, The Dynamic Source Routing protocol (DSR) for mobile ad hoc networks for IPv4, IETF Draft, 2007.
[4] Federal Highway Administration, Highway Statistics 2004, U.S. Department of Transportation.

[5] Information Society Technologies (IST), Dynamic Radio for IP-services in Vehicular Environments (DRiVE), Available at http://wwwistdrive.org/

[6] C. Lochert et al., A routing strategy for vehicular ad hoc networks in city environments, Proceedings of IEEE Intelligent Vehicles Symposium 2003, pp. 156-161.

[7] V. Namboodiri, M. Agarwal and L. Gao, A study on the feasibility of mobile gateways for vehicular ad-hoc networks, Proceedings of ACM VANET'2006, pp. 66-75.

[8] V. Naumov, R. Baumann and T. Gross, An evaluation of inter-vehicle ad hoc networks based on realistic vehicular traces, Proceedings of ACM MobiHoc'2006, pp. 108-119.

[9] V. Naumov and T. R. Gross, Connectivity-aware routing (CAR) in vehicular ad hoc networks, Proceedings of IEEE Infocom'2007, pp. 19191927.

[10] OPNET Technologies, Inc., OPNET Modeler, Available at http://www.opnet.com

[11] D. Sormani et al., Towards lightweight information dissemination in inter-vehicular networks, Proceedings of ACM VANET'2006, pp. 20-29.

[12] W. Su, S-J Lee, and M. Gerla, Mobility prediction and routing in ad hoc wireless networks, Journal of Network Management, Vol. 11, 2001, pp. 3-30.

[13] U.S. Geological Survey, Available at http://www.usgs.gov/

[14] Yahoo!Maps, Available at http://maps.yahoo.com/

[15] M. Zhang and R. S. Wolff, Geographic information based mobility model for mobile ad hoc routing in sparse and rural areas, Proceedings of IEEE Autonet Workshop, 2006.

[16] J. Zhao and G. Cao, VADD: Vehicle-Assisted Data Delivery in vehicular ad hoc networks, Proceedings of IEEE Infocom'2006. 